\title{
Defect interactions on solid surfaces
}

\author{
J.M. Rickman and D.J. Srolovitz \\ Department of Materials Science and Engineering, University of Michigan, Ann Arbor, MI 48109-2136, USA
}

Received 19 August 1992; accepted for publication 20 October 1992

\begin{abstract}
A unified approach to the calculation of the elastic interaction between defects on a surface that describe, for example, steps, islands, and stress domains is presented. This is accomplished by determining the spatial dependence of the elastic displacement fields generated by the idealized point and line forces representing these defects and applying a power counting argument. This argument is then extended to determine the dependence of the interaction energy of two such elastic defects on their separation. In order to apply this analysis to a spatially extended surface defect we develop a multipole expansion of the displacement field that can be used to easily classify the force distribution of this defect in terms of its moments and, hence, determine the dependence of defect-defect interaction energies on separation. As a specific example, we calculate the elastic interaction between two separated circular islands on the surface of an elastic half-space and interpret the results in terms of power counting arguments.
\end{abstract}

\section{Introduction}

A nominally flat surface may contain inhomogeneities or defects such as steps, adatoms, islands, domains of different reconstructions, etc. The origin of surface steps may be traced to statistical fluctuations in the surface profile or the tilting of a solid surface with respect to the underlying bulk crystal planes (i.e., a vicinal surface). The presence of adatoms may be attributed to surface adsorption via an equilibrium vapor, through non-equilibrium deposition or as a result of surface segregation from the bulk of the material. Both Volmer-Weber and Stranski-Krastonov growth produce islands. Domains of different surface reconstructions are thermodynamically preferred in two phase regions of the surface phase diagram. In many cases, a single reconstruction may have several degenerate orientations, including, for example, the well studied $(2 \times 1)$ surface reconstruction of $\mathrm{Si}(001)$, resulting in a reconstructed domain structure. These are but a small selection of possible surface inhomogeneities.

These defects can interact with each other by two distinct mechanisms: through contact and through interactions mediated through the crystal. Hard contact occurs when the defects directly impinge upon one another. Another type of contact, soft contact, occurs through the interaction of the diffusional fields around the defects (e.g., islands on a substrate). The defect-defect interaction that is mediated through the bulk may be traced to the elastic fields a surface defect introduces in the underlying crystal. Since all surface defects have an associated elastic field, this elastic defect-defect interaction is always present. Although the high energy associated with broken bonds (e.g., along steps) or a misfit (e.g., in heteroepitaxy) dominates the defect-defect elastic interactions in determining the surface defect energy, it is the defect-defect interactions that dictate the spatial arrangement of the surface defects and hence controls the surface microstructure. It is this elastic interaction between surface defects that is the focus of the present study.

The effects of these elastically mediated interactions among defects on both the structural properties and the energetics of solids have been addressed in several recent studies [1-5]. For example, the stress and strain fields associated with a periodically stepped surface were found to decay exponentially into 
the bulk, leading to spatial variations in interplanar spacing (i.e., surface relaxation) [1] and a step-step interaction energy that varies inversely with the square of the step separation [1,2]. Further, it has been found that two atoms adsorbed on an elastically isotropic substrate interact through the substrate with an energy that varies inversely with the cube of their separation [3]. The existence of a strain field associated with misfitting islands on a substrate can affect the morphology of the surface as these islands may grow and possibly cluster [4]. The formation of a single orientation of the $(2 \times 1)$ reconstruction of the $\mathrm{Si}(001)$ surface upon application of an external stress has been attributed to the elastic interaction of the strain field of surface domain boundaries with the macroscopic stress field [5].

In general, surface defects can be regarded as entities which exert a distribution of forces on a surface. For instance, a single step behaves as a line of dipole forces acting primarily perpendicular to the step and within the plane of the surface. This force dipole is simply a balance between the surface tensions (stresses) of the two terraces that meet at the step [6,2]. A second contribution to the force distribution around a step may be associated with the large inward relaxation of the most underbound atoms at the top of the step [7] compared with those at the bottom of the step - resulting in another line of force dipoles parallel to the step but directed normal to the plane of the surface $[1,8]$. The distribution of forces associated with a misfitting island may be characterized as a line of forces wrapped around the perimeter of the island. These forces along the island's perimeter are in the plane of the surface and have signs determined by the sign of the island misfit with respect to the surface. As islands are finite in extent, far from the island these forces appear as radial, point force dipoles. In the case of a reconstructed surface consisting of a periodic arrangement of alternating stress domains, there is a discontinuity in the stress at the domain boundaries and, therefore, a force distribution directed normal to the domain boundary and in the plane of the surface [5].

The relationship between a force on the surface and the elastic displacement field in the underlying crystal may be determined from the appropriate elastic influence or Green function. Thus, it should be possible to make rather general arguments about the spatial dependence of elastic fields generated by defects and defect-defect interaction energies from a knowledge of the properties of the force distributions that are involved. In fact, it is simply the spatial (multipole) moments of these force distributions that determine the elastic fields and defect-defect interactions. In short, one goal of the present study is to determine, a priori, the interactions between any two surface defects without having to actually perform a detailed calculation. We will reproduce all previous results of surface defect interactions known to us (e.g., step-step interactions) and generalize the results to those cases not yet studied (e.g., interaction between a misfitting island and a surface step) by using a consistent formalism.

This paper is organized as follows. In section 2 we consider the elastic fields generated by zero- and one-dimensional force distributions localized on the surface of an isotropic, elastic half-space and develop a simple argument that relates the multipole character and spatial dimension of the defect force distributions to the spatial dependence of the associated elastic fields and defect-defect interaction energies. In the following section, we apply the multipole analysis to the case of two separated circular islands on a surface and then compare the results with a nominally exact solution to this problem. Finally, we present results for the spatial dependence of the interactions between a wide variety of surface defects. An appendix is included which shows how to apply the present method to the general case of a spatially extended surface defect.

\section{Elastic fields of surface defects}

In order to analyze the elastic fields generated by a surface defect, we first need to establish the nature of the surface force distribution produced by the defect. This force distribution can be thought of as a source creating the field. There is an excellent analogy between the force (source) and elastic field in 
elasticity and the charges (source) and electrostatic potential in classical electromagnetic theory. The electrostatic potential set up by distribution of charges, depends on the multipole character of the charge distribution. In the theory of elasticity the role of the source is played by the force distribution, and we conclude that a similar multipole analysis will be useful in characterizing elastic fields. An arbitrarily complex force distribution can be exactly described by a spatial distribution of elastic multipoles. Far from the source of these forces, only the lowest order multipoles are important and, hence, from a knowledge of the nature of the fields that are produced by prototypical point and line multipoles the elastic fields and the interaction energy between separated force distributions may be calculated in this limit. However, because of the vector nature of the forces in the theory of elasticity and the boundary conditions associated with the surface geometry, the elasticity analysis is somewhat more complicated than the corresponding analysis in electrostatics. Teodosiu [9] has made extensive use of multipoles in his analyses of point defects in bulk crystals.

In order to calculate the displacement field $u(r)$ associated with a surface force distribution $f(r)$, where $r$ is a two-dimensional surface vector, we start with a surface Green tensor $\bar{G}\left(r, r^{\prime}\right)$ that describes the displacement field at $r$ generated by a point force $r^{\prime}$ on the surface of the solid. In the following development, we shall employ the Green tensor for the elastically isotropic half-space described by $z>0$ and bounded by a free surface which is the $x-y$ plane [10]. We note, that while the Green tensor is appropriate for a flat surface, nominally flat surfaces of real materials may be rough. Surface irregularities produce "image" modifications to the Green tensor. However, provided that the Green tensor is employed to describe elastic effects over length scales large compared with that of the irregularities, the present approach remains valid.

Before using the explicit form for $\bar{G}$, however, it is useful to consider two general properties. First, due to translational invariance in the $x-y$ plane, $\bar{G}\left(r, r^{\prime}\right)=\bar{G}\left(r-r^{\prime}\right)$. Second, the components of the displacement field can be calculated from the relation

$$
u_{\alpha}(r)=\iint \mathrm{d}^{2} r^{\prime} G_{\alpha \beta}\left(r-r^{\prime}\right) f_{\beta}\left(r^{\prime}\right)
$$

where the integration is to be performed over the $x-y$ plane. Hence, the components of the displacement field associated with the point force $f_{\beta}(r)=p_{\beta} \delta(r)$ with strength $p$ are, then, $u_{\alpha}(r)=p_{\beta} G_{\alpha \beta}(r)$.

\subsection{Line multipoles}

As a specific application of eq. (1), we first examine the displacement field produced by a linear distribution of forces on the surface $(z=0)$ acting along the $y$-axis. A convenient way to classify this distribution is in terms of its multipole moments as a complete knowledge of the multipole moments is equivalent to knowing the distribution. With this in mind, consider the prototypical linear force distribution $f_{\beta}(r)=p_{\beta} \delta^{(m)}(x)$, where $(m)$ means the $m$ th spatial derivative and $p_{\beta}$ is a strength (in units of force [length] $\left.{ }^{m-1}\right)$. $m$ refers to the order of the multipole - for example, $m=1$ corresponds to a dipole, $m=2$ to a quadrupole, and, in general, $m$ corresponds to a $2^{m}$ pole. The spatial multipole moments of this force distribution $F_{m}$ are given by

$$
F_{m}=\int_{-\infty}^{\infty} \mathrm{d} x x^{n} f_{\beta}(x)=(-1)^{m} p_{\beta}\left(\begin{array}{c}
n \\
m
\end{array}\right) m ! \delta_{m, n}
$$

and so this $f_{\beta}(r)$ is completely characterized by its $m$ th multipole moment. This $f_{\beta}(r)$ will be referred to here as a linear $m$-pole by analogy with electromagnetic theory. The relation between these $m$-poles and specific defects such as steps and stress domains will be discussed below. 
The displacement field associated with $f_{\beta}(r)$ can now be calculated from eqs. (1) and (2) by

$$
u_{\alpha}(x)=p_{\beta} \iint \mathrm{d}^{2} r^{\prime} G_{\alpha \beta}\left(r-r^{\prime}\right) \delta^{(m)}\left(x^{\prime}\right)=p_{\beta} \frac{\partial^{m}}{\partial x^{m}} \int_{-\infty}^{\infty} \mathrm{d} s G_{\alpha \beta}\left(x^{\prime}, s\right),
$$

where, in the above relation, we have made use of the properties of derivatives of distributions [11]. The result that $\boldsymbol{u}$ is independent of $\boldsymbol{y}$ is due to the translational invariance of the source in the $y$-direction. Although the integral in eq. (3) may be evaluated explicitly, it is possible to draw some general conclusions from the structure of $G_{\alpha \beta}(r)$. Since $G_{\alpha \beta}(r) \alpha(1 / r) g_{\alpha \beta}(\phi)$, where $r=\left(x^{2}+y^{2}\right)^{1 / 2}$ and the $g_{\alpha \beta}(\phi)$ are angular-dependent functions [10], one can see that

$$
\int_{-\infty}^{\infty} \mathrm{d} s G_{\alpha \beta}\left(x^{\prime}, s\right)=A_{\alpha \beta} \ln (x / a)+B_{\alpha \beta},
$$

where the coefficients $A_{\alpha \beta}$ and $B_{\alpha \beta}$ depend on the elastic constants and $a$ is a short-distance cut-off. Upon substituting the explicit form for $G_{\alpha \beta}[10]$ into eq. (4) we find that

$$
\begin{aligned}
& A_{\alpha \beta}=\left(\frac{-2\left(1-\nu^{2}\right)}{\pi E}\right) \delta_{\alpha \beta}-\left(\frac{2 \nu(1+\nu)}{\pi E}\right) \delta_{\alpha 2} \delta_{\beta 2}, \\
& B_{11}=-B_{22}=\left(\frac{2 \nu(1+\nu)}{\pi E}\right), \quad B_{31}=-B_{13}=\left(\frac{(1-2 \nu)(1+\nu)}{2 E}\right),
\end{aligned}
$$

where $E$ is the Young's modulus, $\nu$ is Poisson's ratio and all other $B_{\alpha \beta}$ are zero. After performing the required differentiation in eq. (3) we find that

$$
u_{\alpha}(x)= \begin{cases}A_{\alpha \beta} p_{\beta} \ln (x / a)+B_{\alpha \beta} p_{\beta}, & m=0 ; \\ A_{\alpha \beta} p_{\beta}(-1)^{m-1}(m-1) !(1 / x)^{m} & m \neq 0 .\end{cases}
$$

Hence, from eq. (6) it is clear that the order of the pole that characterizes the linear force distribution and, hence, the type of surface defect determines the dependence of the displacement field on $x$ [12].

By using similar arguments, the interaction energy $e$ (per unit length) between two distinct linear distributions of force may be calculated (i.e., the interaction between a line $m$-pole and a parallel line $n$-pole separated by a distance $d$ ). This can be done by using a simple virtual work argument, which states that the interaction energy $e$ is simply the work that is done in inserting the force distribution of defect (1) into the displacement field generated by defect (2). Thus,

$$
e(d)=-\int_{-\infty}^{\infty} \mathrm{d} x f_{\alpha}(x-d) u_{\alpha}(x)
$$

and so, from eq. (3),

$$
e(d)=\left.(-1)^{n+1} p_{\alpha}^{(1)} p_{\beta}^{(2)} \frac{\partial^{m+n}}{\partial x^{m+n}}\left(\int_{-\infty}^{\infty} \mathrm{d} s G_{\alpha \beta}\left(x-x^{\prime}, s\right)\right)\right|_{x-x^{\prime}=d} .
$$

Then, by employing the general result embodied in eq. (4), one finds that

$$
e(d)= \begin{cases}-\tilde{A} \ln (d / a)-\tilde{B}, & m=n=0 ; \\ (-1)^{m}(m+n-1) ! \tilde{A}(1 / d)^{m+n}, & \text { otherwise }\end{cases}
$$

where $\tilde{A} \equiv A_{\alpha \beta} p_{\alpha}^{(1)} p_{\beta}^{(2)}$ and $\tilde{B} \equiv B_{\alpha \beta} p_{\alpha}^{(1)} p_{\beta}^{(2)}$.

Hence, except for the special case $m=n=0$, the dependence of $e(d)$ on $d$ is described by a power law. The order of the poles that characterize the two linear force distributions determine the dependence 
of $e(d)$ on $d$. This result can be understood by examining eq. (8) and making the following power counting argument. Differentiating $(1 / d) m+n$ times (the sum of the orders of the poles) yields a result proportional to $(1 / d)^{m+n+1}$. But, because we are considering line force distributions, an integration along the line (over $y$ ) remains. This additional integration means that $e \sim\left(C_{1} \ln d+C_{2}\right)\left(\delta_{m, 0} \delta_{n, 0}\right)$ for the special case of $m=n=0$ and $e \sim(1 / d)^{m+n}$ otherwise, where $C_{1}$ and $C_{2}$ are constants.

As a concrete application of these results, we consider the following well known cases: the elastic interaction between surface steps [13] and the interactions between stress domain walls [5]. As described above, a line dipole distribution may be associated with a step [13] - i.e., each step corresponds to a $m=1$ multipole. In this case, we find $e(d)=2\left(1-\nu^{2}\right)\left(p_{1}^{(1)} p_{1}^{(2)}+p_{3}^{(1)} p_{3}^{(2)}\right) /\left(\pi E d^{2}\right)$. Therefore, the displacement field of each step decays as $1 / x$ (eq. (6)) and the interaction energy between two identical steps decays with their separation $d$ as $+1 / d^{2}$ (eq. (9)). The $p_{1}$ terms are associated with in-plane forces and $p_{3}$ with normal forces. If the two parallel steps have opposite sign (i.e., one steps up and the other down), then the $p_{1}^{(1)} p_{1}^{(2)}$ term remains unchanged and the $p_{3}^{(1)} p_{3}^{(2)}$ term changes sign and hence the interaction may be either repulsive or attractive. A stress domain wall such as the domain wall separating a region of $(2 \times 1)$ reconstruction on the Si(001) surface from a region of $(1 \times 2)$ reconstruction, may be described as a line force monopole $(m=0)$ neglecting steps [5]. The interaction energy associated with two opposite sign stress domain walls (which, for example, bound a single $(2 \times 1)$ or $(1 \times 2)$ domain) decays as $-\ln (d)$, i.e. these two domain walls are repulsive. Eq. (9) predicts that the interaction between a stress domain wall and a surface step decays as $1 / d$, where the sign depends on the sign of the step or domain wall. The dependence of $e(d)$ on $d$ for these (and other) cases is summarized below in table 1 . A complete analysis of these types of interactions requires knowledge of $f$. Since $f$ is determined on the atomic scale, we believe that atomistic simulations are required to predict $\hat{A}$ and hence the magnitude and/or sign of the defect interactions.

\subsection{Point multipoles}

The same type of power counting argument can also be made for elastic point poles. Unlike the case of line multipoles discussed above, it is, in general, difficult to construct general expressions for the displacement field and the interaction energy for point poles as the corresponding force distributions may involve higher-order tensors. In order to avoid these complications we consider, as a specific example, the case of a two-dimensional point dipole which has a force distribution

$$
f_{\alpha}(r)=C_{\alpha \beta} \frac{\partial \delta(r)}{\partial x_{\beta}}
$$

where $C_{\alpha \beta}$ are the components of a second-rank tensor [13]. This distribution can describe, for example, an adsorbed impurity atom [3] or a distant misfitting island on a surface. In general, the components of the force distribution of a two-dimensional point $m$-pole will involve terms like $\delta^{(m)}(x) \delta^{(n-m)}(y)$, where $m \leq n$. By integrating this force distribution against the elastic Green tensor, as in eq. (3), one finds that $u$ will involve terms of the form

$$
\iint \mathrm{d}^{2} r^{\prime} G_{\alpha \beta}\left(r-r^{\prime}\right) \delta^{(m)}\left(x^{\prime}\right) \delta^{(n-m)}\left(y^{\prime}\right)=\left.(-1)^{m}\left(\frac{\partial^{n} G_{\alpha \beta}\left(x-x^{\prime}, y-y^{\prime}\right)}{\partial x^{\prime m} \partial y^{\prime n-m}}\right)\right|_{x^{\prime}=y^{\prime}=0}
$$

Since $G_{\alpha \beta}\left(r-r^{\prime}\right)$ scales as $1 /\left|r-r^{\prime}\right|$, differentiating $n$-times (for a $2^{n}$-pole) shows that $u(r) \propto(1 / r)^{n+1}$. This is analogous to the line pole result, except that in the point pole case there is no integration along the line and hence $u$ scales as $1 / r^{n+1}$ instead of as $1 / r^{n}$. It then follows that the interaction energy $E(d)$ between a point $m$-pole and a point $n$-pole that are separated by a distance $d$ is given by

$$
E \propto(1 / d)^{m+n+1}
$$


Table 1

The dependence of the elastic interaction energy for two distinct surface defects separated by a distance $d$

\begin{tabular}{lllll}
\hline & $D=0, m=0$ & $D=0, m=1$ & $D=1, m=0$ & $D=1, m=1$ \\
\hline$D=0, m=0$ & $d^{-1}$ & $d^{-2}$ & $\ln (d), C$ & $d^{-1}$ \\
$D=0, m=1$ & $d^{-2}$ & $d^{-3}$ & $d^{-1}$ & $d^{-2}$ \\
$D=1, m=0$ & $\ln (d), C$ & $d^{-1}$ & $\ln (d), C$ & $d^{-1}$ \\
$D=1, m=1$ & $d^{-1}$ & $d^{-2}$ & $d^{-1}$ & $d^{-2}$ \\
\hline
\end{tabular}

$D$ refers to the spatial dimension of the defect and $m$ refers to its multipole character. For the cases where $D=1$ for both defects, the interaction energy is $e(d)$, the energy per unit defect length. Otherwise the interaction energy is $E(d)$, the total interaction energy. For cases where the interaction energy does not have a power law behavior, it depends on either $\ln (d)$ or $C$ (a constant) depending upon the relative orientation of the defects. The cases considered are a point force $(D=0, m=0)$, an impurity atom or island $(D=0, m=1)$, a stress domain $(D=1, m=0)$ and a step $(D=1, m=1)$.

Eq. (12) suggests that the interaction energy associated with two adsorbed atoms (surface points dipoles) varies as $1 / d^{3}$.

\subsection{Point multipole / line multipole interactions}

Before summarizing the results of the multipole analysis, we consider the mixed case of a point $m$-pole interacting with a line $n$-pole. The interaction energy between the point and line poles can be determined by inserting the displacement field for the point pole and the force distribution for the line pole (or vice versa) into eq. (7). Performing this operation and integrating along the line yields

$$
E \propto \begin{cases}C_{1} \ln (d / a)+C_{2}, & m=n=0 ; \\ (1 / d)^{m+n}, & \text { otherwise; }\end{cases}
$$

where $C_{1}$ and $C_{2}$ are constants.

\subsection{Summary of results}

From the preceding general considerations it is evident that the spatial dependence of elastic fields associated with surface defects and the dependence of defect-defect interaction energies on separation are determined both by the dimensionality and the multipole character of the defects. In terms of the specific surface defects considered here one can now state, for example, the dependence of the interaction energy $E$ on the (large) separation $d$ for the following representative pairs of surface defects: two misfitting islands $\left(d^{-3}\right)$, a misfitting island and an isolated step $\left(d^{-2}\right)$, and an adsorbed atom and a stress domain wall $\left(d^{-1}\right)$. Other combinations of defects are also possible. These results are summarized in table 1.

While the multipole analysis presented above predicts the spatial dependence of the displacement fields and interaction energies for both point and line-like surface defects, many surface defects (such as islands) are not truly point or line-like. Nonetheless, the present analysis yields the appropriate decay of the displacement field and interaction energy at distances large compared with the actual spatial extent of the defect. In this limit, the detailed shape of the surface defect is immaterial. This may be stated more rigorously by noting that, especially for widely separated defects, the lowest-order moments of the force distributions that describe these defects determine their interaction energies. In order to demonstrate the validity of these assertions, we consider the general casc of extended force distributions in the appendix. This analysis does indeed confirm these expectations and so, in order to determine the dependence of defect-defect interaction energies on separation for widely-separated spatially extended defects, one should first calculate the multipole moments associated with each defect and then use the 


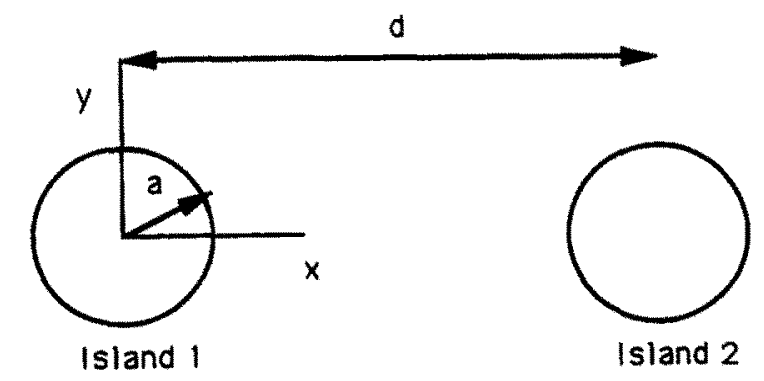

Fig. 1. Two interacting circular islands, each of radius $a$, that are centered on the origin and displaced a distance $d$ along the positive $x$-axis, respectively.

power counting arguments (or, equivalently, table 1). Since the results in the appendix are formal, we consider a simpler extended force distribution in the next section. In particular, we obtain exact results for the displacement field of a circular island and approximate results for the energy of two interacting circular islands of finite extent. These results are then compared with those found by the simple power counting arguments.

\section{Misfitting circular islands - an example}

As an illustration of the previous analysis, we now consider the case of two misfitting circular islands with equal radii on the surface of the half-space that can interact elastically through the medium. This situation is shown schematically in fig. 1. In order to determine the interaction energy for large island separation, we first calculate the moments of the appropriate force distribution for a single island and then use the power counting arguments. We then employ the results found in the appendix to calculate the displacement field due to a single island exactly as well as the (approximate) interaction energy between islands.

The continuous radial force distribution associated with an isolated, misfitting circular island of radius $a$ centered on the origin of the $x-y$ plane is modelled by

$$
f(r)=p \delta(r-a) \hat{r},
$$

where $p$ is the force per unit circumferential length, $r=\left(x^{2}+y^{2}\right)^{1 / 2}$, and $\hat{r}=\hat{\boldsymbol{i}}(x / r)+\hat{j}(y / r)$. In this case we have chosen to neglect any forces normal to the plane. The magnitude of $p$ is determined by the ratio of the lattice parameter of the island to that of the substrate (i.e., by the misfit strain) and by the elastic moduli of the island. The sign of $p$ is determined by the sign of the misfit and is radially outward (inward) for positive (negative) misfit strain. The (cylindrical) multipole moments, $\boldsymbol{M}_{\boldsymbol{m}}$, associated with a circular island, may be determined from the relation

$$
M_{m}=\int \mathrm{d} A^{\prime}\left(r^{\prime}\right)^{m} \mathrm{e}^{-\mathrm{i} m \phi^{\prime}} f\left(\boldsymbol{r}^{\prime}\right),
$$

which, for a given $m$, is a linear combination of the Cartesian moments of order $m$. The primes in the integrand in eq. (15) refer to the coordinates of the force distribution. Inserting eq. (14) into eq. (15) we find that

$$
M_{m}=\pi p a^{m+1} \delta_{m, 1} \epsilon_{-} \quad(m \geq 0),
$$

where $\epsilon_{-}=\hat{i}-\mathrm{i} \hat{j}$ describes a right circularly polarized field.

Since the only non-vanishing multipole coefficient is $\boldsymbol{M}_{1}$ it is expected, from section 2 , that $u \sim(a / r)^{2}$ to lowest order in $(a / r)$, a dipole field. From the circular symmetry of the forces it is also apparent that 
$u \| \hat{r}$ and is radially outward (inward) for $p>0(<0)$. So, from the power counting argument developed in section 2 (see table 1), it is expected that the interaction energy $E \sim d^{-3}$ for two circular islands separated by a distance $d$ that is much larger than $a$. The sign of the interaction energy can also be determined with this information. Since the displacement fields decay with increasing separation, the strongest interaction occurs for the point on island 2 closest to island 1 . The force associated with this point on island 2 and the displacement field due to island 1 (at the position of that point on island 2) are parallel (antiparallel) for $p^{(1)} / p^{(2)}<0(>0)$, where $p^{(1)}$ and $p^{(2)}$ are the forces per unit length associated with islands 1 and 2 , respectively. Hence, like islands repel. This same type of argument can always be used to determine the sign of the interaction between two surface defects. Care must be taken, however, to determine the points on the surface defects where the interaction is maximum. We emphasize here that, for simplicity, we have not considered forces normal to the surface. The inclusion of such forces might change the sign of the interaction in the case of unlike islands.

In order to see this more explicitly, we now use the results of the appendix. Since $f_{\perp}=\hat{\boldsymbol{k}}(\boldsymbol{f} \cdot \hat{\boldsymbol{k}})=\mathbf{0}$ here, it is only necessary to calculate $u_{\|}=\hat{i} u_{x}+\hat{j} u_{y}$ in order to calculate the interaction energy between islands. The spatial dependence of $\boldsymbol{u}_{\|}$can be characterized by calculating the multiple coefficients in eqs. (A.7) and (A.8). One finds that

$$
\begin{aligned}
\boldsymbol{X}_{m}(r) & =p \int \mathrm{d} A^{\prime}\left(r^{\prime}\right)^{m} \mathrm{e}^{-\mathrm{i} m \phi^{\prime}}{ }_{2} F_{1}\left(m+\frac{1}{2}, \frac{1}{2} ; m+1 ;\left(r^{\prime} / r\right)^{2}\right) \delta\left(r^{\prime}-a\right) \hat{\boldsymbol{r}} \\
& =\pi p a_{2}^{m+1} F_{1}\left(m+\frac{1}{2}, \frac{1}{2} ; m+1 ;(a / r)^{2}\right) \delta_{m, 1} \epsilon_{-} \quad(m \geq 0)
\end{aligned}
$$

and

$$
\begin{aligned}
Y_{m}(r) & =p \int \mathrm{d} A^{\prime}\left(r^{\prime}\right)^{m+1} \mathrm{e}^{-\mathrm{i} m \phi^{\prime}}{ }_{2} F_{1}\left(m+\frac{1}{2}, \frac{1}{2} ; m+1 ;\left(r^{\prime} / r\right)^{2}\right) \delta\left(r^{\prime}-a\right) \\
& =2 \pi p a^{m+2}{ }_{2} F_{1}\left(m+\frac{1}{2}, \frac{1}{2} ; m+1 ;(a / r)^{2}\right) \delta_{m, 0},
\end{aligned}
$$

where $F$ is a hypergeometric function. As noted in the appendix, $X_{m} \approx M_{m}$ in the limit that $(r / a) \gg 1$. Since the only non-vanishing multipole coefficients are $X_{1}$ and $Y_{0}$ it follows, again from the appendix, that $\boldsymbol{u}_{\|} \sim(a / r)^{2}$ to lowest order in $(a / r)$, a dipole field. $\boldsymbol{u}_{\|}$can be obtained by substituting the results from eqs. (17) into eqs. (A.5) and (A.6) and then using eq. (A.2a) to yield

$$
u_{i}=\hat{\boldsymbol{r}}\left(\frac{\left(1-\nu^{2}\right)}{E}\right)\left(\frac{4 p}{\pi}\right)\left(\frac{a}{r}\right)^{2} D\left(\frac{a}{r}\right),
$$

where we have used the relationship between the hypergeometric functions, their derivatives and the complete elliptical integrals $D$ and $K$ [14] to simplify the expressions.

A connection can now be made with the previous power counting argument. Expanding $\boldsymbol{D}$ in powers of $(a / r)$ we find that eq. (18) reduces to

$$
u_{\|}=\hat{r}\left[\frac{\left(1-\nu^{2}\right)}{E} p\left(\frac{a}{r}\right)^{2}+\mathrm{O}\left(\left(\frac{a}{r}\right)^{4}\right)\right]
$$

This result may be directly compared with the result of the power counting argument which suggest that $u \propto 1 / r^{2}$. This demonstrates that for $r \gg a$, the power counting result and the exact result coincide.

The interaction energy, $E$, between the two circular islands, one centered on the origin and one displaced by $d=\hat{i} d$, can be calculated from the expression

$$
E=-\int \mathrm{d} A f_{\|}(r-d) \cdot u_{\|}(r) \approx \pi p^{(1)} p^{(2)} a\left(\frac{\left(1-\nu^{2}\right)}{E}\right)\left(\frac{a}{d}\right)^{3},
$$


where, for simplicity, only the leading order term for $u_{\|}$was used in this approximation. From eq. (20) it can be seen that the elastic interaction energy is repulsive for islands with like sign misfit and attractive otherwise. This is the result obtained earlier from geometrical considerations and power counting arguments. The $(1 / d)^{3}$ behavior of $E$ is characteristic of a dipole-dipole interaction energy, as can be seen from table 1.

\section{Conclusions}

It has been shown that, from the nature of the force distribution associated with surface defects, it is possible to make rather general arguments as to the spatial dependence of the associated elastic fields and the dependence of defect-defect interaction energies on separation. This was done by first considering the displacement fields associated with point and line defects and associating these defects with prototypical force distributions. From a general argument based upon the spatial dependence of the Green function that describes the elastic medium, a power counting argument was developed to predict the elastic displacement field of these defects and the energy associated with their interactions. It was found that a surface defect can be classified in terms of its (multipole) moments and that the spatial dependence of the fields generated by the defect and the dependence of defect-defect interaction energies on separation are determined by the lowest-order multipoles. Thus, by knowing that, for example, a step can be viewed as a line of dipoles and an impurity atom as a point dipole, one can determine the nature of their elastic interaction.

This analysis was applied to the case of extended surface defects, in general, and interacting circular islands on the surface of an elastic medium, in particular. These defects were classified in terms of the multipole moments of their associated force distributions and then also described by a power counting argument. Although only the case of an elastically isotropic medium was considered in this paper, it is important to note that the nature of our conclusions as to the relation between the properties of the defects and their associated fields is unchanged for the case of an anisotropic medium. That is, the same power counting arguments apply to the case of an isotropic solid, although the angular dependence of the fields will, in gencral, be more complex. The interaction between defects described by multipoles has essentially the same power law dependence on defect separation in the bulk as it does on the surface due to the fact that the bulk and surface Green tensor have a $1 / r$ dependence.

\section{Acknowledgements}

We gratefully acknowledge the support of the Defense Advanced Research Projects Agency (DARPA) and the Office of Naval Research under Contract N00014-91-J-4019. We also wish to thank R. Najafabadi and J.P. Hirth for many helpful discussions, and I. Rubinstein for motivating this work.

\section{Appendix}

In this appendix, a general relation between the spatial moments of the force distribution that describes a surface defect and the associated displacement field will be derived. Before proceeding with the analysis of the fields set-up by a (possibly) extended source, we first decompose the source and the resulting displacement field as is customary, into in-plane and transverse (i.e., normal to the surface) components. That is,

$$
f=f_{\|}+f_{\perp}, \quad u=u_{\|}+u_{\perp},
$$


where $f_{\|}=\hat{i} f_{x}+\hat{j} f_{y}$ is the in-plane component and $f_{\perp}=\hat{k} f_{z}$ is the transverse component. The same decomposition was used for $\boldsymbol{u}=\boldsymbol{u}_{\|}+\boldsymbol{u}_{\perp}$. Upon substituting the Green tensor for an elastically isotropic half-space [10] into eq. (1), the displacement field due to the force distribution can be written as

$$
\begin{aligned}
\boldsymbol{u}_{\|}= & \left(\frac{\left(1-\nu^{2}\right)}{\pi E}\right) \int \mathrm{d} A^{\prime} \frac{f_{\|}\left(\boldsymbol{r}^{\prime}\right)}{\left|\boldsymbol{r}-\boldsymbol{r}^{\prime}\right|}+\left(\frac{\nu(1+\nu)}{\pi E}\right)\left[\int \mathrm{d} A^{\prime} \frac{f_{\|}\left(\boldsymbol{r}^{\prime}\right)}{\left|\boldsymbol{r}-\boldsymbol{r}^{\prime}\right|}\right. \\
& \left.-\nabla\left(\int \mathrm{d} A^{\prime} \frac{f_{\|}\left(\boldsymbol{r}^{\prime}\right) \cdot\left(\boldsymbol{r}-\boldsymbol{r}^{\prime}\right)}{\left|\boldsymbol{r}-\boldsymbol{r}^{\prime}\right|}\right)\right]-\left(\frac{(1+\nu)(1-2 \nu)}{2 \pi E}\right) \nabla\left(\int \mathrm{d} A^{\prime} f_{\perp}\left(\boldsymbol{r}^{\prime}\right) \cdot \hat{k} \ln \left|\boldsymbol{r}-\boldsymbol{r}^{\prime}\right|\right), \\
\boldsymbol{u}_{\perp}= & \left(\frac{\left(1-\nu^{2}\right)}{\pi E}\right) \int \mathrm{d} A^{\prime} \frac{f_{\perp}\left(\boldsymbol{r}^{\prime}\right)}{\left|\boldsymbol{r}-\boldsymbol{r}^{\prime}\right|}+\left(\frac{(1+\nu)(1-2 \nu)}{2 \pi E}\right) \hat{k} \nabla \cdot\left(\int \mathrm{d} A^{\prime} f_{\|}\left(\boldsymbol{r}^{\prime}\right) \ln \left|\boldsymbol{r}-\boldsymbol{r}^{\prime}\right|\right),
\end{aligned}
$$

where the integration is over the planar surface, $E$ is Young's modulus, $\nu$ is Poisson's ratio, $\left|r-r^{\prime}\right|=$ $\left[\left(x-x^{\prime}\right)^{2}+\left(y-y^{\prime}\right)^{2}\right]^{1 / 2}$, and $\nabla=\hat{i}(\partial / \partial x)+\hat{j}(\partial / \partial y)$, a two-dimensional gradient.

In order to deal with the case of circular islands in section 3 , we first consider the special case of in-plane forces $\left(f_{\perp}=0\right)$. A multipole expansion of the in-plane displacement field, $u_{\|}$, can be obtained by expanding the $1 /\left|r-r^{\prime}\right|$ in the integrand. A useful expansion in circular cylindrical coordinates is [15]

$$
\frac{1}{\left|r-r^{\prime}\right|}=\sum_{m=-\infty}^{\infty} \int_{0}^{\infty} \mathrm{d} k J_{m}(k r) J_{m}\left(k r^{\prime}\right) \mathrm{e}^{\mathrm{i} m\left(\phi-\phi^{\prime}\right)}
$$

where $J_{m}$ is a Bessel function of order $m$. Eq. (A.3) can be rewritten, upon carrying out the $k$-integration [14], as

$$
\frac{1}{\left|r-r^{\prime}\right|}=\operatorname{Re} \sum_{m=0}^{\infty} \frac{\left(2-\delta_{m, 0}\right) \Gamma\left(m+\frac{1}{2}\right)}{\Gamma(m+1) \Gamma\left(\frac{1}{2}\right)} \mathrm{e}^{\mathrm{i} m\left(\phi-\phi^{\prime}\right)}\left(\frac{r_{<}^{m}}{r_{>}^{m+1}}\right){ }_{2} F_{1}\left(m+\frac{1}{2}, \frac{1}{2} ; m+1 ;\left(r_{<} / r_{>}\right)^{2}\right),
$$

where $r_{<}\left(r_{>}\right)$is the smaller (larger) of $r$ and $r^{\prime},{ }_{2} F_{1}\left(m+\frac{1}{2}, \frac{1}{2} ; m+1 ;\left(r^{\prime} / r\right)^{2}\right)$ is a hypergeometric function and $\operatorname{Re}$ means the real part.

Upon substituting eq. (A.4) into eq. (A.2a) one finds that $\boldsymbol{u}_{\|}$can be generated from the following two types of terms:

$$
\begin{aligned}
& \Lambda(r, \phi) \equiv \sum_{m=0}^{\infty} \frac{\mathrm{e}^{\mathrm{i} m \phi}}{r^{m+1}} \frac{\left(2-\delta_{m, 0}\right) \Gamma\left(m+\frac{1}{2}\right)}{\Gamma(m+1) \Gamma\left(\frac{1}{2}\right)} X_{m}(r), \\
& \Psi(r, \phi) \equiv \sum_{m=0}^{\infty} \frac{\mathrm{e}^{\mathrm{i} m \phi}}{r^{m+1}} \frac{\left(2-\delta_{m, 0}\right) \Gamma\left(m+\frac{1}{2}\right)}{\Gamma(m+1) \Gamma\left(\frac{1}{2}\right)} Y_{m}(r),
\end{aligned}
$$

where

$$
\begin{aligned}
& X_{m}(r)=\int \mathrm{d} A^{\prime}\left(r^{\prime}\right)^{m} \mathrm{e}^{-\mathrm{i} m \phi^{\prime}}{ }_{2} F_{1}\left(m+\frac{1}{2}, \frac{1}{2} ; m+1 ;\left(r^{\prime} / r\right)^{2}\right) f\left(r^{\prime}\right), \\
& Y_{m}(r)=\int \mathrm{d} A^{\prime}\left(r^{\prime}\right)^{m} \mathrm{e}^{-\mathrm{i} m \phi^{\prime}}{ }_{2} F_{1}\left(m+\frac{1}{2}, \frac{1}{2} ; m+1 ;\left(r^{\prime} / r\right)^{2}\right)\left(f\left(r^{\prime}\right) \cdot r^{\prime}\right),
\end{aligned}
$$

and where $X_{m}(r)$ and $Y_{m}(r)$ are cylindrical multipole coefficients. Eqs. (A.5) and (A.6) apply to the displacement field outside of the force distribution. From eq. (A.2a) it can be seen that $u_{\|}$contains the 
terms $\Lambda, \nabla(r \cdot \Lambda)$, and $\nabla \Psi$, and so the spatial dependence of $u_{\|}(r, \phi)$ is known once the $m$ values that contribute to eqs. (A.5) and (A.6) are determined. The allowed values of $m$ are determined from eqs. (A.7) and (A.8).

Both $X_{m}(r)$ and $Y_{m}(r)$ contain information about the $m$ th moments of the source. In fact, in the limit that $(r / a) \gg 1$, where $a$ is a characteristic dimension of the source, we have that

$$
\boldsymbol{X}_{m}(r) \approx \boldsymbol{M}_{m}=\int \mathrm{d} A^{\prime}\left(r^{\prime}\right)^{m} \mathrm{e}^{-\mathrm{i} m \phi^{\prime}} f\left(\boldsymbol{r}^{\prime}\right)
$$

and similarly for $Y_{m}(r)$. Eq. (A.9) can be obtained by substituting a series expansion for the hypergeometric function [16] into eq. (A.7) and then examining the far-field limit in which the hypergeometric function is, to zeroth order in $(a / r)$, replaced by unity. Thus, the leading behavior of $u_{\|}$is $(1 / r)^{m^{*}+1}$, where $m^{*}$ is the first integer for which either $\boldsymbol{X}_{m}$ or $Y_{m}$ does not vanish.

In order to calculate $u$ it is often necessary to consider those terms in eqs. (A.2a) and (A.2b) with $\ln \left|\boldsymbol{r}-\boldsymbol{r}^{\prime}\right|$ in the integrand. Proceeding as above, we can first expand $\ln \left|\boldsymbol{r}-\boldsymbol{r}^{\prime}\right|$, the two-dimensional electrostatic Green function, as [15]

$$
\ln \left|r-r^{\prime}\right|=\ln r-\sum_{m=1}^{\infty}\left(\frac{1}{m}\right)\left(\frac{r^{\prime}}{r}\right)^{m} \cos \left[m\left(\phi-\phi^{\prime}\right)\right]
$$

and then substitute this expansion into the relevant terms in eqs. (A.2a) and (A.2b). It is again possible to write a multipole expansion analogous to eqs. (A.5) and (A.6) for these terms.

\section{References}

[1] D.J. Srolovitz and J.P. Hirth, Surf. Sci. 255 (1991) 111.

[2] J.H. van der Merwe and G.J. Shiflet, Surf. Sci. 256 (1991) 171.

[3] K.H. Lau and W. Kohn, Surf. Sci. 65 (1977) 607.

[4] Y. Golan, L. Margulis, I. Rubinstein and G. Hodes, to be published.

[5] O.L. Alerhand, D. Vanderbilt, R.D. Meade and J.D. Joannopoulos, Phys. Rev. Lett. 61 (1988) 1973; see also J. Tersoff and E. Pehlke, Phys. Rev. Lett. 68 (1992) 816.

[6] P. Nozières, in: Solids Far From Equilibrium (Cambridge University Press, Cambridge, 1992).

[7] M.W. Finnis and V. Heine, J. Phys. F 4 (1974) L37.

[8] S.P. Chen, A.F. Voter and D.J. Srolovitz, Phys. Rev. Lett. 57 (1986) 1358.

[9] C. Teodosiu, Elastic Models of Crystal Defects (Springer, New York, 1982).

[10] L.D. Landau and E.M. Lifshitz, Theory of Elasticity (Pergamon, New York, 1970).

[11] I. Sneddon, The Use of Integral Transforms (McGraw-Hill, New York, 1972).

[12] More rigorously, one should consider a line force distribution of finite length such as that given by $f_{\beta}\left(r^{\prime}\right)=p_{\beta} \delta^{(m)}\left(x^{\prime}\right) \Theta\left(y^{\prime}-\right.$ $L / 2) \Theta\left(y^{\prime}-2 y+L / 2\right)$, where $\Theta(x)$ is the step function and $L$ is a length. With this modification eq. (4) becomes $f_{y}^{L}-L / z$ d $s G_{\alpha \beta}\left(x^{\prime}, s\right) \approx A_{\alpha \beta}[\ln (x / L)+O(1 / L)]+B_{\alpha \beta}$. Upon differentiating $m(m \geq 0)$ times and taking the limit $L \rightarrow \infty$ one recovers eq. (6) for $m \geq 0$.

[13] V.I. Marchenko and A.Ya. Parshin, Sov. Phys. JETP 52 (1980) 129;

A.F. Andreev and Y.A. Kosevich, Sov. Phys. JETP 54 (1982) 761.

[14] I.S. Gradshteyn and I.M. Ryzhik, Table of Integrals, Series, and Products (Academic Press, New York, 1980).

[15] J.D. Jackson, Classical Electrodynamics (Wiley, New York, 1975).

[16] G.B. Arfken, Mathematical Methods for Physicists (Academic Press, New York, 1970). 